\title{
Eliciting customers' waste reduction and water saving behaviors at a hotel
}

\begin{abstract}
Without a doubt, sustainability is a critical issue in the world's hotel industry. The present study examined the key cognitive, affective, and normative determinants of customers' waste reduction and water saving intentions during their hotel stay. This research also investigated the effect of involvement of green behaviors in everyday life as a moderator. A survey methodology and quantitative approach were utilized while conducting this study. Our results revealed that environmental value, concern, and awareness acted as significant cognitive triggers in forming pro-environmental intentions, and that anticipated feelings were important contributors to increasing intentions. The salient role of moral norm in determining environmentally responsible decision was also uncovered. Moreover, the results from the invariance test demonstrated that involvement of green behaviors included a significant moderating effect. Overall, our conceptual model sufficiently explained the variance in pro-environmental intentions among hotel customers.
\end{abstract}

Keyword: Waste reduction; Water saving; Involvement of green behaviors; Anticipated feelings; Hotel customers 